\title{
Emerging technologies in autoantibody testing for rheumatic diseases
}

\author{
Nancy J. Olsen ${ }^{1 *}$, May Y. Choi ${ }^{2}$ and Marvin J. Fritzler ${ }^{2}$
}

\begin{abstract}
Testing for the presence of antinuclear antibodies (ANAs) is a key step in the diagnosis of systemic lupus erythematosus (SLE) and other systemic autoimmune rheumatic diseases (SARD). The standard slide-based indirect immunofluorescence (IIF) test is widely used, but is limited by a relative lack of specificity for SLE and not all SARD-ANAs are detected. Alternative immunoassays that might offer enhanced diagnostic and prognostic information have evolved, and some of these have entered clinical practice. This review summarizes the current state of ANA testing and multiplex techniques for detecting other autoantibodies, the possibility of point-of-care testing, and approaches for applications in early disease stages.

Keywords: Systemic autoimmune rheumatic diseases, Systemic lupus erythematosus, Antinuclear antibodies, Autoantibodies, Biomarkers, Point of care testing, Immunoassays
\end{abstract}

\section{Background}

Systemic autoimmune rheumatic diseases (SARD) afflict $3-5 \%$ of the population [1] and are associated with enormous burdens on individuals and society due to poor quality of life, lower productivity, and growing direct and indirect healthcare costs [2,3]. A hallmark of SARD is the presence of autoantibodies directed against a spectrum of intracellular autoantigens, in general referred to as antinuclear antibodies (ANAs) [4]. Often, ANAs are the only disease-specific serological markers for an underlying SARD and, as such, many have become part of classification criteria developed to provide a common basis for disease prediction and prognosis, an accurate diagnosis, disease monitoring, entry into

\footnotetext{
*Correspondence: nolsen@pennstatehealth.psu.edu

${ }^{1}$ Penn State M.S. Hershey Medical Center, 500 University Drive, Hershey, PA

17033, USA

Full list of author information is available at the end of the article
}

therapeutic trials, and understanding the pathogenesis of the SARD [4-7]. While the ANA test is generally requested as a means of confirming a diagnosis and intent to treat a specific SARD, in a general practice setting it is also used as an approach to case finding [8]. Although clinicians formulate a differential diagnosis based on history and physical examination, practice analysis shows that most readily act only after receiving confirmatory or exclusionary laboratory test results $[9,10]$. Hence, it is important to have an understanding of the proper use of autoantibody testing and its value, as well as limitations [4].

One of the major challenges in adopting an evidencebased approach to ANA testing is the discrepancies observed in studies that characterize autoantibodies as biomarkers of disease onset and disease activity. These discrepancies are due to a wide range of variables that include technical aspects of clearly defined methodology and assay performance, but are also due to a lack of prospective or longitudinal studies, patient selection bias, use of inconsistent definitions for disease activity, variations in nomenclature of autoantibodies, frequency of testing, and effects of therapy, which contribute to conflicting results $[11,12]$. It should also be noted that disease activity, disease severity, and the ensuing irreversible tissue and organ damage should be conceptually distinguished, and measurement tools for these parameters may be different [13]. Despite these potential problems in interpreting ANA results, the clinician's goal of making an early and accurate diagnosis as well as judging disease activity has made the practice of ordering autoantibodies widespread and frequent $[14,15]$. For example, more than $90 \%$ of US rheumatologists use serial anti-dsDNA autoantibody titers to monitor disease activity in systemic lupus erythematosus (SLE) [10].

In this review, we outline the use of common and emerging technologies that are used to detect antinuclear and related autoantibodies (Table 1). 
Table 1 Advantages of various ANA immunoassay techniques

\begin{tabular}{|c|c|c|c|c|c|c|c|c|c|c|}
\hline Assay Type & Low cost & Rapid & Automated & Sensitivity & Specificity & Quantitative & Availability & Reproducibility & High throughput & $\begin{array}{l}\text { Small sample } \\
\text { volume }\end{array}$ \\
\hline IIF & + & + & $(++)^{*}$ & ++ & + & ++ & ++ & + & + & - \\
\hline ELISA & ++ & ++ & ++ & +++ & ++ & ++ & ++ & ++ & ++ & + \\
\hline LIA & - & ++ & ++ & ++ & ++ & ++ & ++ & + & + & ++ \\
\hline $\begin{array}{l}\text { ALBIA, CIA, other } \\
\text { bead-based arrays }\end{array}$ & ++ & +++ & +++ & +++ & ++ & +++ & ++ & + & +++ & +++ \\
\hline Planar array & ++ & ++ & ++ & ++ & ++ & + & - & - & ++ & ++ \\
\hline Point-of-Care & + & +++ & - & + & ++ & + & - & ++ & - & + \\
\hline
\end{tabular}

*Automated antinuclear antibody (ANA)-indirect immunofluorescence (IIF), antineutrophil cytoplasmic antibody (ANCA), and Crithidia luciliae fluorescent test (anti-dsDNA; CLIFT) used in some laboratories

ALBIA addressable laser bead immunoassays, CIA chemiluminescence immunoassay, ELISA enzyme-linked immunosorbent assay, LIA line immunoassay

\section{Autoantibody detection}

Indirect immunofluorescence (IIF): including automated, digital IIF

Indirect immunofluorescence (IIF) for the detection of ANAs and other autoantibodies has been in use for more than 50 years and the substrates have evolved from rodent organ sections to tissue culture (i.e., HEp-2) cells [4]. The IIF method has the advantages of providing a semiquantitative result, unlike the LE cell preparation that it displaced, and offers insights about other potential antigen reactivities by displaying staining patterns that provide a clue to the specific autoantibody targets $[16,17]$. Although diagnostic technologies have evolved from low-resolution microscopy to highly automated, robotic, and digital microscopy [4, 18], IIF remains one of the most widely used screening tests for SARD [4, 19, 20]. In 2010, due to concerns about other technologies such as enzyme linked immunosorbent assays (ELISAs) and other diagnostic kits that were being increasingly used as the ANA screening test by high-throughput laboratories, the American College of Rheumatology engaged a study group which eventually published a position paper indicating that IIF on HEp-2 cells should remain the "gold standard" for the detection of ANAs [21]. While this might have been expected to encourage diagnostic laboratories to return to the IIF ANA test, there is little evidence that this has happened. Indeed, when an ANA IIF on HEp-2 substrates is used as a screening test for the wide spectrum of SARD, it is troubled by both false positive and false negative results and other limitations that may mislead the clinician $[4,9$, 22]. Despite decades of effort, standardization of the IIF ANAs has been a challenge due to intermanufacturer variations in the production of the HEp-2 substrate, characteristics of the secondary antibody, and expertise and subjectivity of the person(s) performing and reading the slides [20, 23]. In addition, the lack of consensus on ANA pattern nomenclature was a major issue which is now being resolved due to the efforts of the International Consensus on ANA Patterns (ICAP) group [24].
Novel approaches to improving the sensitivity and specificity of the IIF ANA test include both 'knock-in' and 'knock-out' gene technology. In the former, to improve the ability to detect anti-SSA/Ro60 on HEp-2 substrates, Tom Gordon at Flinders University in Adelaide developed a 'knock-in' cell line where the corresponding Ro60 cDNA was transfected and overexpressed in HEp-2 cells [25], an approach that led to the HEp2000 substrate (ImmunoConcepts, Sacramento, CA, USA) that is used in some diagnostic laboratories [26]. More recently, a 'knock-out' substrate has been developed as an approach to detecting antibodies to dense fine speckles (DFS70) antigen (discussed later) during the screening ANA IIF test. In this substrate, 'normal' HEp-2 cells are admixed with HEp-2 cells that have the DFS70 gene knocked out at a ratio of 9:1, allowing detection of DFS70 [27]. It must be emphasized that this approach only detects sera with monospecific anti-DFS70 autoantibodies and still requires wider interlaboratory validation.

\section{ELISA}

ELISAs used for the detection of autoantibodies in SARD sera came into wide use in the 1980s and have become a mainstay in many diagnostic laboratories. In this setting, they have two main uses: 1) as a quantitative screening test to detect a wide variety of ANAs without indicating the specificity of a positive test result; and 2) as an approach to identifying specific autoantibody targets. Standard available ELISA panels used for SARD diagnosis usually include SSA/Ro, SSB/La, Sm, Sm/RNP, Scl-70/topoisomerase I, Jo-1, anti-centromere, and anticardiolipin, and others are increasingly available. ELISA results are reported as quantitative units rather than as serum dilutions as for IIF.

During the last decade, different strategies have been utilized to develop, evaluate, and commercialize several ANA screening ELISAs in an attempt to eliminate the more labor-intensive and subjective ANA IIF assay (reviewed in $[28,29]$ ). The majority of these ANA screening ELISAs make use of blends of purified proteins derived 
from native sources (i.e., cells or tissues) and/or synthetic, recombinant technologies [30]. The composition of these preparations is as diverse as the various manufacturers producing them but, in general, they contain many of the key target autoantigens associated with SARD. Despite remarkable progress, they are attended by technical challenges of combining different autoantigens in a single assay, and reactivity to some SARD-related autoantibody targets can be missed even if the autoantigens are contained in the mixtures [31]. This is probably due to differences in physicochemical properties of individual autoantigens and it is suspected that some antigens also bind to other targets in the same mixture, resulting in a masking effect that may lead to poor autoantibody binding. Although ELISAs that are widely used to detect individual autoantibodies generally perform much better than ELISAs that use antigen mixtures, some limitations of autoantibody binding to exposed epitopes on the autoantigens that are absorbed to the plastic microtiter plates are observed in those assays as well.

\section{Line immunoassays}

Line immunoassays (LIAs) are considered a variation of immunoblots and dot-blots [4]. A wide assortment of LIAs are commercially available and they are typically used to identify not just ANAs, but also other autoantibodies [4, 32, 33]. In some settings, LIAs also have been used to screen for disease-specific autoantibodies that are seen in SARD, paraneoplastic, and autoimmune liver diseases, and one standard kit includes 15 different SARD-related autoantibody specificities [4, 33]. One limitation of LIAs is that they are not as adaptable to high-volume and high-throughput laboratories, although components of the technology have been automated making them more suitable in these settings. The reactivity of autoantibodies to specific LIA targets can be quantitated by densitometry. Despite their ease of use, LIAs have other drawbacks, including the lack of sensitivity and specificity for certain autoantibodies [32, 33].

\section{Addressable laser bead immunoassays}

Addressable laser bead immunoassays (ALBIAs) were introduced approximately 15 years ago as a rapid, economical, quantitative, and reliable technology to detect autoantibodies directed to a range of target autoantigens in SARD sera (reviewed in [34]). Multiplexed bead-based technologies have been adopted by several diagnostic kit manufacturers based on two main technology platforms, Luminex and BioFlash chemiluminescence immunoassay (CIA) [34-38]. Similar to ELISA and LIA kits, ALBIA/ CIAs are used for the detection of autoantibodies to a variety of autoantigens, [39-43] and the number and characteristics of autoantigens employed in ALBIA/CIAs and their performance vary between kit manufacturers.
Hence, it is important to be aware of the performance characteristics of the specific ALBIA/CIA kits used by diagnostic laboratories. A study comparing ALBIA, ANA IIF and different ELISA assays for the detection of ANA found that $7.4 \%$ of healthy donors had a positive test and, of those, the majority had a speckled IIF staining pattern [41], raising the question whether these 'false positive' ANAs detected by IIF might represent autoantibodies to DFS70, a marker that has been shown to be more common in healthy or nonSARD patients than in SARD patients $[44,45]$. A recent study of a large international inception SLE cohort confirmed that isolated anti-DFS70 antibodies using CIAs are rare in SLE (1.1\%) [46]. Therefore, this antibody may be helpful in distinguishing ANApositive individuals and SLE. Of note, the DFS70 nomenclature, also termed lens epithelium-derived growth factor (LEDGF), refers to the IIF staining pattern characterized by uniformly distributed fine speckles throughout the interphase nucleus and on metaphase chromatin along with the autoantigen target having a molecular mass of $70 \mathrm{kDa}$ in immunoblots [47]. Although anti-DFS antibodies were first reported in interstitial cystitis and were later found to be associated with a number of other conditions, including atopic dermatitis, the current consensus is that they rarely occur in isolation in SLE, systemic sclerosis, Sjögren's syndrome, and mixed connective tissue diseases [44].

\section{Multiplexed autoantibody arrays Planar arrays}

SLE is characterized by humoral autoimmunity and over 180 distinct autoantibodies have been associated with the disease [48]. This means that usual laboratory panels of ANA-related autoantibodies, designed to detect 10 to 15 different types, provide a view of less than $10 \%$ of the autoreactivity that may be present in a patient. SLE is therefore an ideal disease for diagnostic approaches using planar arrays that detect hundreds or more different autoantibodies.

Most of these techniques involve robotic spotting of purified autoantigen proteins or selected oligonucleotides on glass slides, although other support matrices have also been used. Investigators at Stanford University first reported, about 15 years ago, studies using an array consisting of 196 molecules corresponding to major autoantigens associated with human diseases [49]. Autoantibodies in human serum that recognize these targets are quantitatively detected by a secondary fluorescent antibody, and results show greater sensitivity than those obtained using standard ELISAs. In a more recent iteration, these investigators have developed a protein microarray with other components that are capable of detecting antibodies to soluble mediators including cytokines [50].

A similar type of array was developed by $\mathrm{Zhu}, \mathrm{Li}$ and colleagues at the University of Texas Southwestern, 
using known autoantigens for various human diseases [51]. Detection of autoantibodies in different classes was shown to be correlated with some types of SLE-related conditions. For example, patients with incomplete forms of lupus, designated as incomplete lupus erythematosus (ILE), were comparable to SLE patients in terms of the total quantity of serum autoreactivity, but relatively more of these autoantibodies were in the IgM class [52]. These array data were analyzed using unbiased clustering programs similar to those employed for gene expression microarrays (Fig. 1). The computer algorithms produced clusters of related autoantibodies, such as ones that reacted with structural or nuclear antigens, providing insights into how autoantibodies might be related to each other. Further insights were developed when the autoantibody array data were correlated with gene signatures expressed in the same ILE or SLE patients. Expression of the Type I interferon (IFN) gene signature that is characteristic of SLE and other autoinflammatory conditions was found to be correlated with high levels of the IgG autoantibodies. This suggests a role for IFN in driving the class switch from IgM to IgG, thus probably contributing to more active disease manifestations in the SLE patients [53].

Related findings were reported by others using a different array that detected 930 autoreactivities, split between IgMs and IgGs [54]. Patients with SLE in this study had upregulation of IgG autoantibodies, while some IgM autoantibodies were distinctly downregulated. This study also showed that SLE patients in remission had autoantibody patterns that were distinct from those of healthy controls. Serendipitously, one healthy control in this study, who initially classified within the SLE domain, subsequently developed an illness that was consistent with SLE, suggesting that the array could detect preclinical stages of the disease.

Insights into genetic effects on the autoantibody profile were provided by studies of SLE twins using arrays detecting 65 autoantigens [55]. The arrays in this analysis provided a panoramic view of IgG and IgM patterns, permitting detection of what were essentially shared IgG fingerprints or signatures in monozygotic SLE twins. While previous studies had suggested this type of association in twins, the scope of autoantibodies detected with the arrays provided the opportunity to establish a much more detailed concordance.

An advantage of the planar array approach is that only very small volumes of serum sample are required to detect hundreds of specificities (Table 1). When a fluid is not available in abundance, and especially for studies designed to explore novel specificities, this feature assumes greater importance. An example is the use of array profiling to study biomarkers in cerebrospinal fluid (CSF) [56]. Using a human proteome microarray with approximately 17,000 proteins, patients with neuropsychiatric (NP)SLE were found to have significantly more autoreactive antibodies in CSF than patients who did not have NPSLE. A network analysis of these data using the Ingenuity Pathway Analysis tool (Ingenuity System Inc, USA) showed that most of these samples were enriched in pathways related to neurologic disease; not a surprising result, perhaps, but

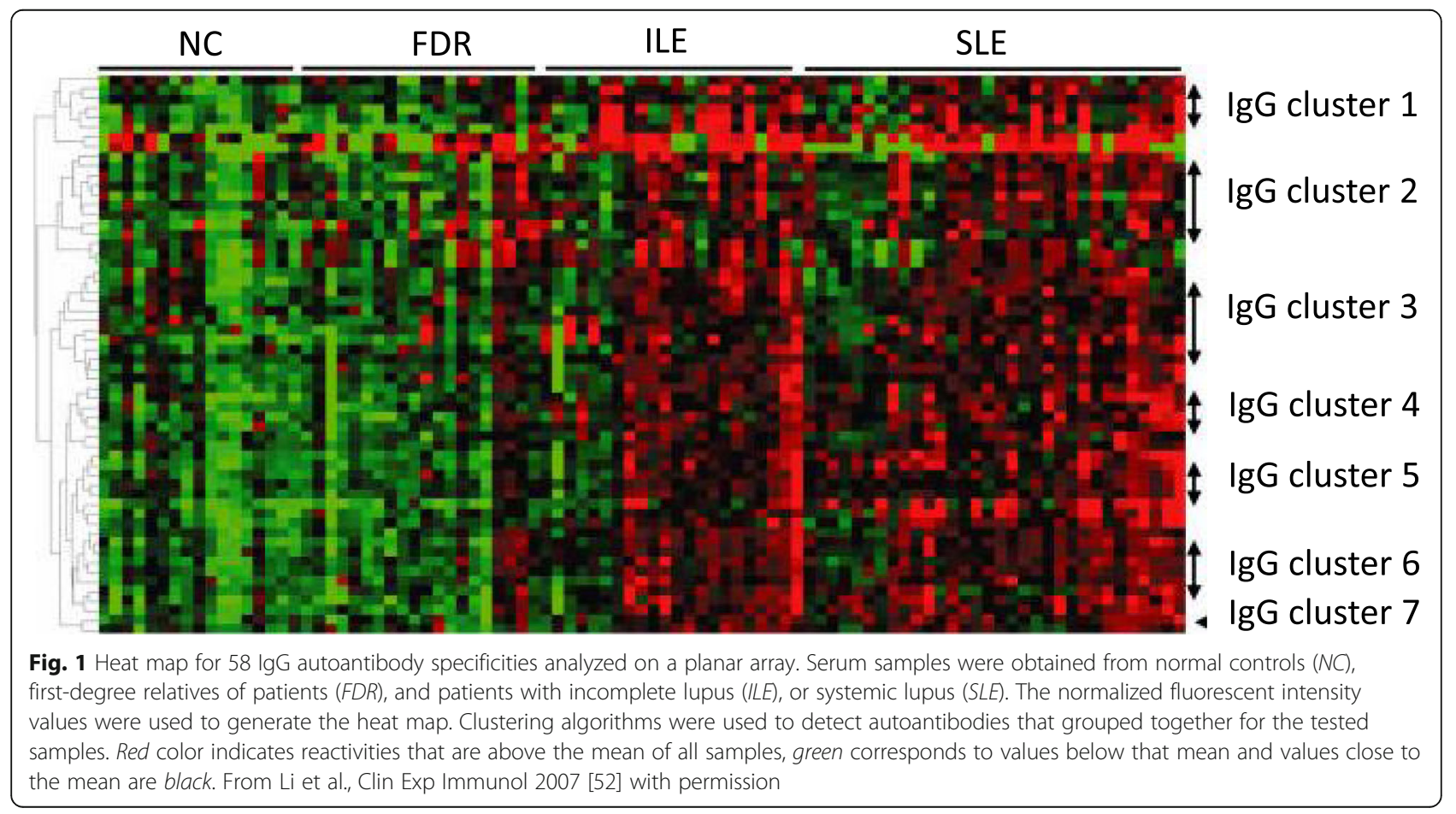


reassuring that the method does detect physiologically relevant specificities. Furthermore, this is another demonstration of how array autoantibodies might be analyzed to give a more complete picture of overall relatedness.

In patients seen in a pediatric setting, detection of large numbers of analytes from small volume samples is also especially useful. An array displaying 140 autoantigens was used to compare pediatric patients with lupus nephritis (LN) to aged-matched healthy controls [57]. Candidate autoantigens for pediatric LN identified by cluster analyses were validated with ELISA. Specificities that were associated with $\mathrm{LN}$ included some that are generally known, such as C1q and dsDNA, but others that are relatively novel, such as collagens IV and X. Previous studies in adult SLE using a different autoantigen array had also shown clustering of collagen IV with dsDNA, suggesting that these independent results have broad validity [52]. Furthermore, the pediatric patients were found to have elevated levels of antibodies to B cell activating factor (BAFF), as had been described previously by this group in adult SLE [50]. The pediatric studies also attempted to develop a predictive signature for LN from a longitudinal subset of the patients, and these data suggest that persistently low nephritis scores derived from array analyses might be associated with a stable course.

Urine is a very relevant biosample to study in SLE, given the high prevalence of renal involvement and the need for sensitive and noninvasive biomarkers of $\mathrm{LN}$. Array analyses of urine samples offer the potential for insights into mediators that may be specific for renal injury. One recent report utilized a commercially available investigational array of 274 human cytokines that is described as being suitable for all liquid sample types [58]. The study included serum and urine samples from SLE patients and the array findings were validated by ELISA. This approach identified adipokines, including adiponectin, resistin, and leptin, as potentially important mediators in LN and utilized pathways analysis to show relationships between different specificities. This illustrates the utility of an array approach for biomarker discovery.

The use of selected autoantigen arrays carries with it the limitation of bias for known specificities. Alternatives to the use of known autoantigens include synthetic ligands such as peptoids which have been used to create highly diverse unbiased libraries that have potential for uncovering novel specificities and biomarkers $[59,60]$. Isolation of IgGs binding to arrayed peptoids in patients with SLE identified autoantibodies to RNA-binding proteins [61]. An unexpected result in this study was that one ligand bound several different autoantibodies, possibly indicating polyreactivity of these autoantibodies. These data generated an estimated specificity for an SLE diagnosis of greater than $97 \%$, with a positive likelihood ratio of 7.45 to 28 .

One array platform that has been developed for clinical applications has the specific objective of excluding of the likelihood of a diagnosis of SLE [62, 63]. The low specificity of ANA testing for SLE makes this a very significant problem for clinical rheumatologists, who are confronted with screening large numbers of referred individuals, only a few of whom actually have disease [8]. A more predictive test would be welcome as a tool to prioritize patients for evaluation. For the SLE exclusion test, an array of 200 specificities, including known autoantigens and a set of proprietary oligonucleotides, was used to generate a dataset from SLE patients and healthy controls that was then analyzed by linear discriminant analysis to optimize sensitivity and specificity. A schematic of the data might be presented to the clinician, showing where the individual tested fits into the profile (Fig. 2). The cutoff chosen for a negative result, meaning SLE is unlikely, performs with a sensitivity of $94 \%$, specificity of $75 \%$, and negative predictive value of $93 \%$. While the separation is imperfect, the test has potential to help overbooked rheumatologists to triage consults for possible lupus evaluations.

The strengths and weaknesses of planar autoantigen arrays have been well described (Table 2) [51, 64]. In general, the unbiased approach to collecting data on a large number of autoantibodies is a major advantage for exploratory studies. Given the relatively small number of autoantibodies detected in clinical diagnostic laboratories compared to the many known antibodies in SLE and other SARD, it is highly likely that new specificities can be found that offer greater utility for successful disease classification and for predicting flares. A major challenge

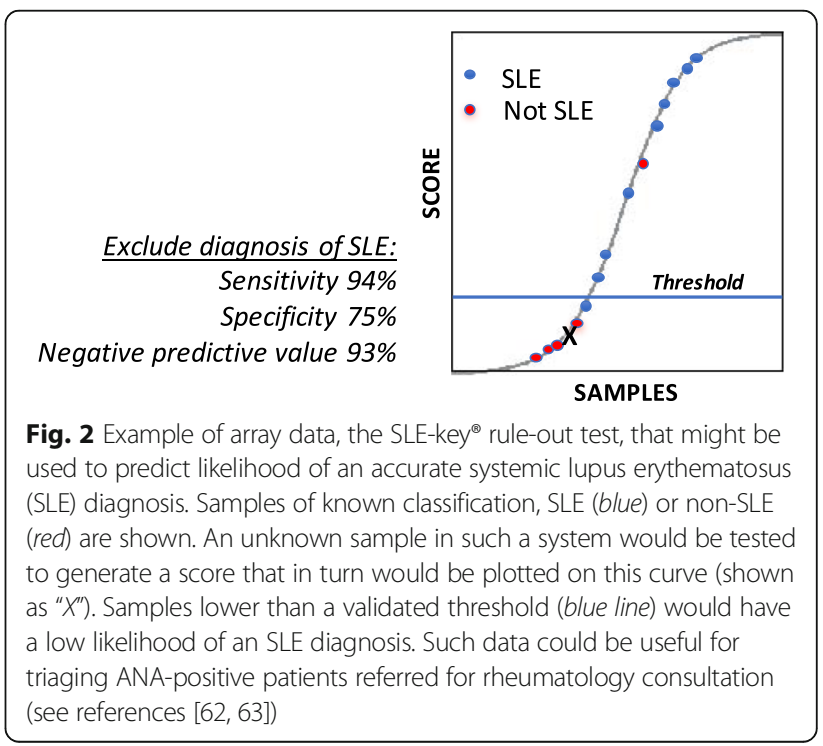


Table 2 Strengths and Weaknesses of Autoantigen Array Methods

\begin{tabular}{ll}
\hline Strengths & Permits screening of many autoantigen targets simultaneously \\
& Uses very small sample volumes \\
Can make use of stored samples & May detect multiple antibody classes or subclasses \\
& Has high throughput capabilities \\
& Permits exploratory approaches to find new targets \\
& Applies unbiased analytics \\
& Carries generally lower cost per specificity than ELISA \\
& Develops insights into autoantibody clusters and relatedness \\
Weaknesses & Potentially difficult to optimize all targets in one array \\
& Has batch-to-batch variability \\
& Generally lacks standardization between laboratories \\
& Normalization standards are variable \\
& Has diminished sensitivity for low-affinity autoantibodies \\
& May miss autoantibodies present at low concentrations \\
& Some autoantigens are not suitable as targets \\
& Results may be semiquantitative
\end{tabular}

is the jurisdictional (i.e., Food and Drug Administration) approval and certification of array assays for routine use in diagnostic laboratories. Clinical diagnostic applications will require reproducibility between different platforms, decreased inter- and intratest variability, standardized quantitation, and interlaboratory validation; the lupus rule-out test discussed above may have overcome some of these obstacles [51, 62].

Detection of rare or low-affinity autoantibodies is another challenge. Addressing these issues likely will require advances in materials science in terms of developing better platforms and detection methods. An example is the use of plasmonic gold substrates with near-infrared fluorescence enhanced imaging, which has been shown to deliver a broader dynamic range and improved performance in detection of low-affinity antibodies compared to results obtained with glass slides [65]. Labeling modifications might include use of twocolor antigen-binding antibody fragments (Fabs), which has been shown to enhance reproducibility of autoantibody detection [66].

\section{Bead-based arrays}

An alternative to the two dimensional or planar array platform is the use of beads to carry the targets, such as those in ALBIAs and CIAs, discussed above. This approach also has been used to produce an 86-specifity assay for selected autoantigens [67]. The target proteins were from commercial sources or were produced in an E. coli expression system. Antibody binding from diluted serum samples was measured using a fluorochromelabeled secondary antibody, and an instrument was used to quantitate fluorescence intensity. Analysis of single autoantibodies demonstrated the expected wide range of prevalence in SLE patients $(n=69)$. These included wellknown associations such as with dsDNA, but also revealed autoantibodies that were not known previously to be associated with SLE. The latter included some related to apoptosis and neutrophil extracellular traps (NETs) which have been shown to contain SLE-related autoantigens [68]. Multivariate analyses showed clear separation of healthy controls from the SLE patients, and clusters of related autoantibodies in the SLE patients were demonstrated using a heat map. A limitation of this study was that other autoimmune diseases or conditions were not included, so performance for distinguishing conditions related to SLE or subsets of SLE such as ILE or LN is unknown. The authors raised the question of whether bead arrays might have less batch-to-batch variability which would make this approach possibly preferable to planar designs for applications in clinical assays, but direct comparisons of bead and planar arrays have not been reported, so this remains speculative. Bead-based arrays do likely have some distinct advantages including reproducibility, rapid and high-throughput design, and reasonable cost.

\section{Detection of early disease}

In the past decade, it has become clear that autoantibodies are present in the preclinical stages of SARD including SLE $[69,70]$. This information has raised interest in pushing the diagnostic window earlier in the time course, prior to the development of significant morbidity or organ damage. Patients with ILE have fewer autoantibodies than SLE patients as well as lower ANA titers [71]. Studies using two-dimensional autoantigen arrays have shown that ILE patients have patterns of autoreactivity that lie intermediate between healthy individuals and those with SLE [52]. Furthermore, ratios of autoantibodies expressed in IgG and IgM classes differ, with the ILE patients showing relatively greater IgM-class autoreactivity. ILE patients are not necessarily individuals who are in early stages of lupus, but ILE is clearly a category that includes patients at high risk of progression to classifiable SLE. In one small study, 3 of 22 patients with ILE who progressed to SLE over a mean time period of 2.4 years showed significant increases in some IgG specificities detected on an array [72]. One of these was SSB, consistent with other findings indicating that this specificity increases late in the preclinical period [73]. Whether something like the SLE exclusion test array that has already been discussed [62] might help to discriminate ILE patients with and without risk of progression is an interesting possibility that has not been investigated.

\section{Point of care (POC) diagnostic technologies}

The consultative and diagnostic services in rheumatology are not typically considered clinical emergencies, which require same-day diagnostic or clinical decisions. While this may hold true for certain chronic and noninflammatory conditions, it is important to appreciate that 
SLE, vasculopathies, and antiphospholipid syndrome can present with or develop intercurrent acute and lifethreatening features which need to be diagnosed and acted upon quickly to prevent irreversible immunemediated damage and mortality. It is in this and other clinical settings that POC testing may be useful [74]. It has been estimated that $10-25 \%$ of all patients with rheumatologic disorders visiting emergency departments require hospital admission, and up to one-third of hospitalized patients need intensive care [75-78]. These emergencies may present as a rapidly evolving multisystem organ failure, as a mimic of other conditions such as an infectious disease, or with misleading, deceptively benign clinical signs. In a clinic setting, a negative result of a POC ANA test might offer immediate insight and eliminate the need for ordering other expensive autoantibody profiles or referral to a specialist (Fig. 3). Similar approaches could apply to acute-onset vasculopathies such as granulomatosis with polyangiitis or renal-pulmonary syndrome where a specific POC test based on proteinase 3 , myeloperoxidase, and/or glomerular basement membrane antibodies might have value as an adjunct to initiating immediate immunotherapy [79]. Having a high level of suspicion, sufficient clinical knowledge, and a method for detection of circulating autoantibody markers are factors that contribute significantly to a timely diagnosis. The use of specific autoantibody testing for the diagnostic process in an acute clinical setting is growing in demand. Availability of a POC for ANA testing has the potential to change practice patterns, not just in rheumatology but also in primary care [80].

New technologies ranging from fairly simple lateral flow technologies to more sophisticated lab-on-a-chip that deliver rapid, low-cost, highly specific, and quantitative results is a goal of point of care testing. Current POC immunoassay technologies come in various configurations and complexities (reviewed in [74, 81]). Several of the newer biosensors have equaled or surpassed traditional central laboratory methods in performance metrics such as sensitivity, specificity, and especially time to result. Devising reliable assays for measuring a specific antibody in human serum is more difficult than measuring most nonantibody analytes in biological fluids, because any one antibody specificity is usually a tiny fraction of total serum immunoglobulin. Nonspecific binding of immunoglobulin may have impeded the development of reliable antibody biosensors. However, recent and evolving advances in the technologies of immunosensors have provided improving accuracy in quantification and low detection limit in testing for some autoantibodies (i.e., anti-dsDNA) commonly used in rheumatology clinical practice $[82,83]$. By virtue of its rapid, bed-side, real-time data collection capability, POC testing has the potential to change the practice of medicine, and rheumatology will not be an exception. Commercialization, regulatory certification, and clinician

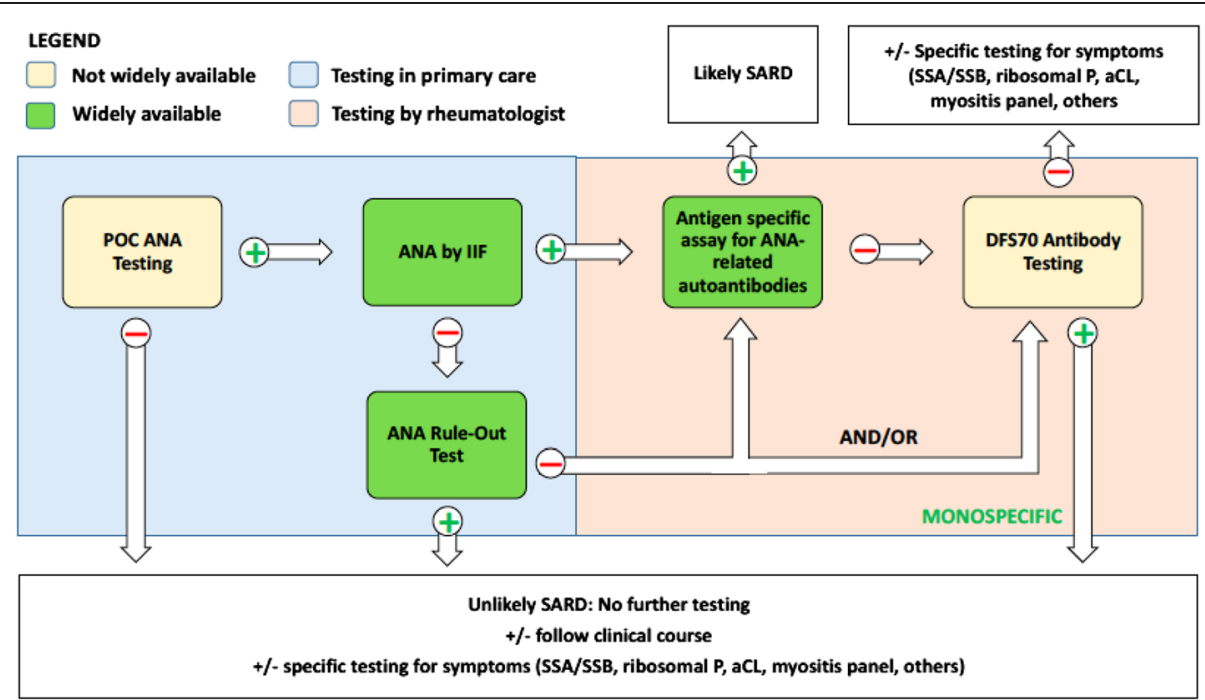

Fig. 3 A schematic of one possible algorithm for evaluation of an SLE diagnosis using existing and proposed technologies. A point of care (POC) test for antinuclear antibodies (ANA) might be used as an office screening tool in primary care. A positive result would prompt further screening by measuring ANA by indirect immunofluorescence (IIF); the SLE-key ${ }^{\circledR}$ rule-out test might be also used. Positivity for ANA and a "not-ruled out" result for the SLE-key ${ }^{\circledast}$ test would then suggest a need for further autoantibody profiling using an antigen-specific assay. A negative test result for ANA, even with a "ruled-out" result on the SLE-key ${ }^{\circledast}$ test, may require further evaluation depending on the clinical scenario. DFS70 testing might be used to further improve diagnostic accuracy. The blue box indicates testing that might be done in primary care, with other tests being done after rheumatology referral. Yellow boxes indicate tests in development or not widely available; green boxes indicate tests that are clinically available, at least in the USA. aCL anti-cardiolipin antibodies, DFS70 dense fine speckles 70, SARD systemic autoimmune rheumatic diseases 
acceptance of POC autoantibody testing in rheumatology is a work in progress.

\section{Other technologies}

A number of other technologies have been developed for the high-throughput testing of sera for autoantibodies. These include a fully automated ANA screening assay [84], a microbead-based ELISA system [85], and nanobarcode technology [86].

\section{Conclusion}

ANA testing has been in clinical use for more than 60 years and was a major advancement in defining SLE as a disease. Measurements of ANAs and related autoantibodies are now commonly used by rheumatologists and nonspecialist providers for diagnosis and classification of SLE and other SARD. However, limitations of the currently available tests have prompted development of other approaches that would offer greater specificity, prognostic value, cost efficiency, and other advantages. These approaches include multiplexed planar and bead arrays as well as rapid detection methods for POC testing, with small sample volumes. Applications including those that would provide assessment of the risk of disease progression are likely to enter clinical practice in the next decade.

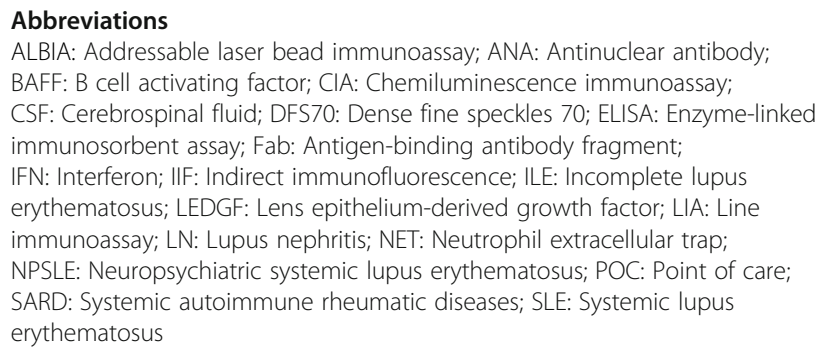

\section{Acknowledgements}

Not applicable.

Funding

Not applicable.

\section{Availability of data and materials}

Not applicable.

\section{Authors' contributions}

NJO and MJF organized the topics for review. NJO, MYC, and MJF contributed equally to the writing and preparation of illustrations. All authors reviewed and approved the final version submitted for publication.

\footnotetext{
Authors' information

NJO is Professor of Medicine and Chief of Rheumatology at the Penn State M.S. Hershey Medical Center in Hershey PA. MYC is a member of the Division of Rheumatology at the University of Calgary, Alberta, Canada. MJF is a professor in the Department of Medicine and Department of Biochemistry
} and Molecular Biology at the University of Calgary.

Ethical approval and consent to participate

Not applicable.
Consent for publication

Not applicable.

\section{Competing interests}

NJO has a research grant from Mallinckrodt Pharmaceuticals and has been a site investigator for Resolve Therapeutics, Aurina Pharmaceuticals, Pfizer, and Horizon Pharmaceuticals. She has been an unpaid consultant for Immunarray Ltd. and IQuity, Inc. MJF is a consultant to Inova Diagnostics Inc. (San Diego, CA, USA), Werfen International (Barcelona, Spain), and has received gifts in kind from Euroimmun GmbH (Luebeck, Germany) and Alexion Pharma Canada. MYC declares that she has no competing interests.

\section{Publisher's Note}

Springer Nature remains neutral with regard to jurisdictional claims in published maps and institutional affiliations.

\section{Author details}

${ }^{1}$ Penn State M.S. Hershey Medical Center, 500 University Drive, Hershey, PA 17033, USA. ${ }^{2}$ Cumming School of Medicine, University of Calgary, Calgary, AB T2N4N1, Canada.

Published online: 24 July 2017

\section{References}

1. Maas K, Chan S, Parker J, Slater A, Moore J, Olsen N, Aune TM. Cutting edge: molecular portrait of human autoimmune disease. J Immunol. 2002;169(1):5-9.

2. Jacobson DL, Gange SJ, Rose NR, Graham NM. Epidemiology and estimated population burden of selected autoimmune diseases in the United States. Clin Immunol Immunopathol. 1997;84(3):223-43.

3. Cooper GS, Stroehla BC. The epidemiology of autoimmune diseases. Autoimmun Rev. 2003;2(3):119-25.

4. Mahler M, Meroni PL, Bossuyt X, Fritzler MJ. Current concepts and future directions for the assessment of autoantibodies to cellular antigens referred to as anti-nuclear antibodies. J Immunol Res. 2014;2014:315179.

5. Valenzuela A, Chung L, Casciola-Rosen L, Fiorentino D. Identification of clinical features and autoantibodies associated with calcinosis in dermatomyositis. JAMA Dermatol. 2014;150(7):724-9.

6. Choi MY, Barber MR, Barber CE, Clarke AE, Fritzler MJ. Preventing the development of SLE: identifying risk factors and proposing pathways for clinical care. Lupus. 2016;25(8):838-49.

7. Olsen NJ, McAloose C, Carter J, Han BK, Raman I, Li QZ, Liao D. Clinical and immunologic profiles in incomplete lupus erythematosus and improvement with hydroxychloroquine treatment. Autoimmune Dis. 2016;2016:8791629.

8. Fritzler MJ. Choosing wisely: review and commentary on anti-nuclear antibody (ANA) testing. Autoimmun Rev. 2016;15(3):272-80.

9. Hu SL, Wang D, Gou WJ, Lei QF, Ma TA, Cheng JZ. Diagnostic value of phospholipase A2 receptor in idiopathic membranous nephropathy: a systematic review and meta-analysis. J Nephrol. 2014;27(2):111-6.

10. Dougherty ER. Biomarker development: prudence, risk, and reproducibility. Bioessays. 2012:34(4):277-9.

11. Kavanaugh AF, Solomon DH. American College of Rheumatology Ad Hoc Committee on Immunologic Testing Guidelines. Guidelines for immunologic laboratory testing in the rheumatic diseases: anti-DNA antibody tests. Arthritis Rheum. 2002;47(5):546-55.

12. Conrad K, Andrade LE, Chan EK, Mahler M, Meroni PL, Pruijn GJ, Steiner G, Shoenfeld $Y$. From autoantibody research to standardized diagnostic assays in the management of human diseases - report of the 12th Dresden Symposium on Autoantibodies. Lupus. 2016;25(8):787-96.

13. Guillemin F. How to assess musculoskeletal conditions. Assessment of disease activity. Best Pract Res Clin Rheumatol. 2003;17(3):415-26.

14. Yazdany J, Schmajuk G, Robbins M, Daikh D, Beall A, Yelin E, Barton J, Carlson A, Margaretten M, Zell J, et al. Choosing wisely: the American College of Rheumatology's top 5 list of things physicians and patients should question. Arthritis Care Res (Hoboken). 2013;65(3):329-39.

15. Kleinnijenhuis J, van der Molen RG, Franssen PM, Berden JH, van der Meer JW, Jacobs JF. Anti-ribosomal P antibodies as a single serological marker in SLE: lupus in disguise. Scand J Rheumatol. 2013;42(2):165-6.

16. Pisetsky DS. Antinuclear antibody testing - misunderstood or misbegotten? Nat Rev Rheumatol. 2017. doi:10.1038/nrrheum.2017.74. 
17. Chan EK, Damoiseaux J, De Melo CW, Carballo OG, Conrad K, Francescantonio PL, Fritzler MJ, Garcia-De La Torre I, Herold M, Mimori T, et al. Report on the second International Consensus on ANA Pattern (ICAP) workshop in Dresden 2015. Lupus. 2016;25(8):797-804.

18. Lakos G, Gonzalez M, Flaherty D, Bentow C, Ibarra C, Stimson D, Nacario L, Hiemann R, Dervieux T. Detection of anti-dsDNA antibodies by computer-aided automated immunofluorescence analysis. J Immunol Methods. 2016;433:17-22.

19. Nossent $H$, Rekvig OP. Antinuclear antibody screening in this new millennium: farewell to the microscope? Scand J Rheumatol. 2001;30(3): 123-6. discussion 127-8.

20. Fritzler MJ. The antinuclear antibody test: last or lasting gasp? Arthritis Rheum. 2011;63(1):19-22.

21. Meroni $P$, Schur P. ANA screening: an old test with new recommendations. Ann Rheum Dis. 2010;69(8):1420-2.

22. Kavanaugh A, Tomar R, Reveille J, Solomon DH, Homburger HA. Guidelines for clinical use of the antinuclear antibody test and tests for specific autoantibodies to nuclear antigens. American College of Pathologists. Arch Pathol Lab Med. 2000;124(1):71-81.

23. Mahler M, Ngo JT, Schulte-Pelkum J, Luettich T, Fritzler MJ. Limited reliability of the indirect immunofluorescence technique for the detection of anti-RibP antibodies. Arthritis Res Ther. 2008;10(6):R131.

24. Damoiseaux J, von Mühlen CA, Garcia-De La Torre I, Carballo OG, de Melo Cruvinel W, Francescantonio PL, Fritzler MJ, Herold M, Mimori T, Satoh M, et al. International consensus on ANA patterns (ICAP): the bumpy road towards a consensus on reporting ANA results. Auto Immun Highlights. 2016;7(1):1.

25. Keech CL, McCluskey J, Gordon TP. Transfection and overexpression of the human 60-kDa Ro/SS-A autoantigen in HEp-2 cells. Clin Immunol Immunopathol. 1994;73(1):146-51.

26. Fritzler MJ, Hanson C, Miller J, Eystathioy T. Specificity of autoantibodies to SS-A/Ro on a transfected and overexpressed human $60 \mathrm{kDa}$ Ro autoantigen substrate. J Clin Lab Anal. 2002;16(2):103-8.

27. Malyavantham K, Suresh L. Analysis of DFS70 pattern and impact on ANA screening using a novel HEp-2 ELITE/DFS70 knockout substrate. Auto Immun Highlights. 2017:8(1):3.

28. Sinclair D, Saas M, Williams D, Hart M, Goswami R. Can an ELISA replace immunofluorescence for the detection of anti-nuclear antibodies? The routine use of anti-nuclear antibody screening ELISAs. Clin Lab. 2007;53(3-4):183-91.

29. López-Hoyos M, Rodríguez-Valverde V, Martinez-Taboada V. Performance of antinuclear antibody connective tissue disease screen. Ann N Y Acad Sci. 2007;1109:322-9.

30. Fenger M, Wiik A, Høier-Madsen M, Lykkegaard JJ, Rozenfeld T, Hansen MS, Samsoe BD, Jacobsen S. Detection of antinuclear antibodies by solid-phase immunoassays and immunofluorescence analysis. Clin Chem. 2004;50(11):2141-7.

31. Copple SS, Sawitzke AD, Wilson AM, Tebo AE, Hill HR. Enzyme-linked immunosorbent assay screening then indirect immunofluorescence confirmation of antinuclear antibodies: a statistical analysis. Am J Clin Pathol. 2011;135(5):678-84

32. Almeida González D, Cabrera de León A, Rodríguez Pérez MC, Brito Díaz B, González Hernández A, García García D, Vázquez Moncholi C, Aguirre Jaime A. Efficiency of different strategies to detect autoantibodies to extractable nuclear antigens. J Immunol Methods. 2010;360(1-2):89-95.

33. Lee SA, Kahng J, Kim Y, Park YJ, Han K, Kwok SK, Park SH, Oh EJ Comparative study of immunofluorescent antinuclear antibody test and line immunoassay detecting 15 specific autoantibodies in patients with systemic rheumatic disease. J Clin Lab Anal. 2012;26(4):307-14.

34. Fritzler MJ, Fritzler ML. Microbead-based technologies in diagnostic autoantibody detection. Expert Opin Med Diagn. 2009;3(1):81-9.

35. Mahler M, Bentow C, Serra J, Fritzler MJ. Detection of autoantibodies using chemiluminescence technologies. Immunopharmacol Immunotoxicol. 2016;38(1):14-20.

36. Ghillani P, Rouquette AM, Desgruelles C, Hauguel N, Le Pendeven C, Piette JC, Musset L. Evaluation of the LIAISON ANA screen assay for antinuclear antibody testing in autoimmune diseases. Ann N Y Acad Sci. 2007;1109:407-13.

37. Zafrir Y, Gilburd B, Carrasco MG, Kivity S, Sánchez-Castañón M, López-Hoyos M, Mandel M, Szmyrka M, Shoenfeld Y, Agmon-Levin N. Evaluation of an automated chemiluminescent immunoassay kit for antinuclear antibodies in autoimmune diseases. Immunol Res. 2013;56(2-3):451-6.

38. Xu X, Wu WY, Tu WZ, Chu HY, Zhu XX, Liang MR, Xue Y, Wang JC, Zou HJ. Increased expression of S100A8 and S100A9 in patients with diffuse cutaneous systemic sclerosis. A correlation with organ involvement and immunological abnormalities. Clin Rheumatol. 2013;32(10):1501-10.
39. Shovman O, Gilburd B, Barzilai O, Shinar E, Larida B, Zandman-Goddard G, Binder SR, Shoenfeld Y. Evaluation of the BioPlex 2200 ANA screen: analysis of 510 healthy subjects: incidence of natural/predictive autoantibodies. Ann N Y Acad Sci. 2005;1050:380-8.

40. Shovman O, Gilburd B, Zandman-Goddard G, Yehiely A, Langevitz P, Shoenfeld Y. Multiplexed AtheNA multi-lyte immunoassay for ANA screening in autoimmune diseases. Autoimmunity. 2005;38(1):105-9.

41. Avaniss-Aghajani E, Berzon S, Sarkissian A. Clinical value of multiplexed bead-based immunoassays for detection of autoantibodies to nuclear antigens. Clin Vaccine Immunol. 2007;14(5):505-9.

42. Hanly JG, Su L, Farewell V, Fritzler MJ. Comparison between multiplex assays for autoantibody detection in systemic lupus erythematosus. J Immunol Methods. 2010:358(1-2):75-80.

43. Nifli AP, Notas G, Mamoulaki M, Niniraki M, Ampartzaki V, Theodoropoulos PA, Kopnitsky MJ, Castanas E. Comparison of a multiplex, bead-based fluorescent assay and immunofluorescence methods for the detection of ANA and ANCA autoantibodies in human serum. J Immunol Methods. 2006;311(1-2):189-97.

44. Mahler M, Meroni PL, Andrade LE, Khamashta M, Bizzaro N, Casiano CA Fritzler MJ. Towards a better understanding of the clinical association of anti-DFS70 autoantibodies. Autoimmun Rev. 2016;15(2):198-201.

45. Seelig CA, Bauer O, Seelig HP. Autoantibodies against DFS70/LEDGF exclusion markers for systemic autoimmune rheumatic diseases (SARD). Clin Lab. 2016;62(4):499-517.

46. Choi MY, Clarke AE, St Pierre Y, Hanly JG, Urowitz MB, Romero-Diaz J, Gordon C, Bae SC, Bernatsky S, Wallace DJ, et al. The prevalence and determinants of anti-DFS70 autoantibodies in an international inception cohort of systemic lupus erythematosus patients. Lupus. 2017. doi:10.1177/ 0961203317692437.

47. Ochs RL, Mahler M, Basu A, Rios-Colon L, Sanchez TW, Andrade LE, Fritzler MJ, Casiano CA. The significance of autoantibodies to DFS70/LEDGFp75 in health and disease: integrating basic science with clinical understanding. Clin Exp Med. 2016;16(3):273-93.

48. Yaniv G, Twig G, Shor DB, Furer A, Sherer Y, Mozes O, Komisar O, Slonimsky

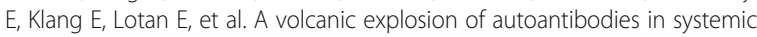
lupus erythematosus: a diversity of 180 different antibodies found in SLE patients. Autoimmun Rev. 2015;14(1):75-9.

49. Robinson WH, DiGennaro C, Hueber W, Haab BB, Kamachi M, Dean EJ, Fournel S, Fong D, Genovese MC, de Vegvar HE, et al. Autoantigen microarrays for multiplex characterization of autoantibody responses. Nat Med. 2002;8(3):295-301.

50. Price JV, Haddon DJ, Kemmer D, Delepine G, Mandelbaum G, Jarrell JA, Gupta R, Balboni I, Chakravarty EF, Sokolove J, et al. Protein microarray analysis reveals BAFF-binding autoantibodies in systemic lupus erythematosus. J Clin Invest. 2013;123(12):5135-45.

51. Zhu H, Luo H, Yan M, Zuo X, Li QZ. Autoantigen microarray for highthroughput autoantibody profiling in systemic lupus erythematosus. Genomics Proteomics Bioinformatics. 2015;13(4):210-8.

52. Li Q, Zhou J, Wandstrat A, Carr-Johnson F, Branch V, Karp D, Mohan C, Wakeland $\mathrm{E}$, Olsen N. Protein array autoantibody profiles for insights into systemic lupus erythematosus and incomplete lupus syndromes. Clin Exp Immunol. 2007;147(1):60-70.

53. Li Q, Zhou J, Lian Y, Zhang B, Branch V, Carr-Johnson F, Karp D, Mohan C, Wakeland $\mathrm{E}$, Olsen $\mathrm{N}$. Interferon signature gene expression is correlated with autoantibody profiles in patients with incomplete lupus syndromes. Clin Exp Immunol. 2010;159(3):281-91.

54. Fattal I, Shental N, Mevorach D, Anaya JM, Livneh A, Langevitz P, Zandman-Goddard G, Pauzner R, Lerner M, Blank M, et al. An antibody profile of systemic lupus erythematosus detected by antigen microarray. Immunology. 2010;130(3):337-43.

55. Silverman G, Srikrishnan R, Germar K, Goodyear C, Andrews K, Ginzler E, Tsao B. Genetic imprinting of autoantibody repertoires in systemic lupus erythematosus patients. Clin Exp Immunol. 2008;153(1):102-16.

56. Hu C, Huang W, Chen H, Song G, Li P, Shan Q, Zhang X, Zhang F, Zhu H, $\mathrm{Wu} L$, et al. Autoantibody profiling on human proteome microarray for biomarker discovery in cerebrospinal fluid and sera of neuropsychiatric lupus. PLoS One. 2015;10(5):e0126643.

57. Haddon DJ, Diep VK, Price JV, Limb C, Utz PJ, Balboni I. Autoantigen microarrays reveal autoantibodies associated with proliferative nephritis and active disease in pediatric systemic lupus erythematosus. Arthritis Res Ther. 2015;17:162. 
58. Hutcheson J, Ye Y, Han J, Arriens C, Saxena R, Li QZ, Mohan C, Wu T. Resistin as a potential marker of renal disease in lupus nephritis. Clin Exp Immunol. 2015;179(3):435-43.

59. Fritzler MJ. Toward a new autoantibody diagnostic orthodoxy: understanding the bad, good and indifferent. Autoimmunity Highlights. 2012;3(2):51-8.

60. Lindstrom TM, Robinson WH. Fishing for biomarkers with antigen mimics. Cell. 2011;144(1):13-5.

61. Quan J, Lakhanpal A, Reddy MM, Zaman S, Li QZ, German DC, Olsen NJ, Kodadek T, Karp DR. Discovery of biomarkers for systemic lupus erythematosus using a library of synthetic autoantigen surrogates. J Immunol Methods. 2013;402(1-2):23-34.

62. Cohen IR, Putterman C, Jordan N, Jakobi K, Sorek R, Blumenstein Y, Batty S SLE-key Rule-out serologic test for SLE using the immunarray ICHIP. Annals Rheum Dis. 2015;74(Suppl 2)

63. Putterman C, Wu A, Reiner-Benaim A, Batty DS, Sanz I, Oates J, Jakobi K Petri M, Safer P, Gerwien R, et al. SLE-key $\left({ }^{\oplus}\right)$ rule-out serologic test for excluding the diagnosis of systemic lupus erythematosus: developing the ImmunArray iCHIP( $\left.{ }^{(}\right)$. J Immunol Methods. 2016;429:1-6.

64. Wang L, Mohan C, Li QZ. Arraying autoantibodies in SLE-lessons learned. Curr Mol Med. 2015;15(5):456-61.

65. Zhang B, Jarrell JA, Price JV, Tabakman SM, Li Y, Gong M, Hong G, Feng J, Utz PJ, Dai H. An integrated peptide-antigen microarray on plasmonic gold films for sensitive human antibody profiling. PLoS One. 2013;8(7):e71043.

66. Kattah MG, Alemi GR, Thibault DL, Balboni I, Utz PJ. A new two-color Fab labeling method for autoantigen protein microarrays. Nat Methods. 2006; 3(9):745-51.

67. Budde P, Zucht HD, Vordenbäumen S, Goehler H, Fischer-Betz R, Gamer M, Marquart K, Rengers P, Richter J, Lueking A, et al. Multiparametric detection of autoantibodies in systemic lupus erythematosus. Lupus. 2016;25(8):812-22.

68. Pisetsky DS. Anti-DNA antibodies-quintessential biomarkers of SLE. Nat Rev Rheumatol. 2016;12(2):102-10.

69. Arbuckle M, McClain M, Rubertone M, Scofield R, Dennis G, James J, Harley $J$. Development of autoantibodies before the clinical onset of systemic lupus erythematosus. N Engl J Med. 2003;349(16):1526-33.

70. Olsen NJ, Karp DR. Autoantibodies and SLE: the threshold for disease. Nat Rev Rheumatol. 2014;10(3):181-6.

71. Aberle T, Bourn RL, Munroe ME, Chen H, Roberts VC, Guthridge JM, Bean K, Robertson JM, Sivils KL, Rasmussen A, et al. Clinical and serological features distinguish patients with incomplete lupus classification from systemic lupus erythematosus patients and controls. Arthritis Care Res (Hoboken). 2017. doi:10. 1002/acr.23201.

72. Olsen NJ, Li QZ, Quan J, Wang L, Mutwally A, Karp DR. Autoantibody profiling to follow evolution of lupus syndromes. Arthritis Res Ther. 2012;14(4):R174.

73. Lu R, Munroe ME, Guthridge JM, Bean KM, Fife DA, Chen H, Slight-Webb SR, Keith MP, Harley JB, James JA. Dysregulation of innate and adaptive serum mediators precedes systemic lupus erythematosus classification and improves prognostic accuracy of autoantibodies. J Autoimmun. 2016;74:182-93.

74. Fritzler MJ. Perspectives on the imperatives, opportunities and challenges for point of care diagnostics in systemiclupus erythematosus. Int J Clin Rheumatol. 2015;9:449-56.

75. Janssen NM, Karnad DR, Guntupalli KK. Rheumatologic diseases in the intensive care unit: epidemiology, clinical approach, management, and outcome. Crit Care Clin. 2002;18(4):729-48.

76. Sharma M, Leirisalo-Repo M. Arthritis patient as an emergency case at a university hospital. Scand J Rheumatol. 1997;26(1):30-6.

77. Panopalis P, Gillis JZ, Yazdany J, Trupin L, Hersh A, Julian L, Criswell LA, Katz $P$, Yelin E. Frequent use of the emergency department among persons with systemic lupus erythematosus. Arthritis Care Res (Hoboken). 2010;62(3):401-8.

78. Hanly JG, Thompson K, Skedgel C. Utilization of ambulatory physician encounters, emergency room visits, and hospitalizations by systemic lupus erythematosus patients: a 13-year population health study. Arthritis Care Res (Hoboken). 2016;68(8):1128-34

79. Offermann N, Conrad K, Fritzler MJ, Fooke AM. Development and validation of a lateral flow assay (LFA) for the determination of IgG-antibodies to Pr3 (cANCA) and MPO (pANCA). J Immunol Methods. 2014;403(1-2):1-6.

80. Konstantinov KN, Rubin RL. The universe of ANA testing: a case for point-of-care ANA testing. Auto Immun Highlights. 2017;8(1):4

81. Konstantinov KN, Tzamaloukas A, Rubin RL. Detection of autoantibodies in a point-of-care rheumatology setting. Auto Immun Highlights. 2013;4(2):55-61.
82. Rubin RL, Wall D, Konstantinov KN. Electrochemical biosensor for quantitation of anti-DNA autoantibodies in human serum. Biosens Bioelectron. 2014;51:177-83.

83. Poulsen NN, Pedersen ME, Østergaard J, Petersen NJ, Nielsen CT, Heegaard $\mathrm{NH}$, Jensen $\mathrm{H}$. Flow-induced dispersion analysis for probing anti-dsDNA antibody binding heterogeneity in systemic lupus erythematosus patients: toward a new approach for diagnosis and patient stratification. Anal Chem. 2016;88(18):9056-61.

84. Hayashi N, Kawamoto T, Mukai M, Morinobu A, Koshiba M, Kondo S, Maekawa S, Kumagai S. Detection of antinuclear antibodies by use of an enzyme immunoassay with nuclear HEp-2 cell extract and recombinant antigens: comparison with immunofluorescence assay in 307 patients. Clin Chem. 2001:47(9):1649-59.

85. Grossmann K, Roggenbuck D, Schröder C, Conrad K, Schierack P, Sack U Multiplex assessment of non-organ-specific autoantibodies with a novel microbead-based immunoassay. Cytometry A. 2011;79(2):118-25.

86. Smith J, Onley D, Garey C, Crowther S, Cahir N, Johanson A, Painter S, Harradence G, Davis R, Swarbrick P. Determination of ANA specificity using the UltraPlex platform. Ann N Y Acad Sci. 2005;1050:286-94. 